\title{
Theoretical Study on Electronic Structure and Optical Properties of Phenothiazine-Containing Conjugated Oligomers and Polymers
}

\author{
Li Yang ${ }^{1}$, Ji-Kang Feng ${ }^{1,2^{*}}$, Ai-Min Ren ${ }^{1}$ \\ 1. State Key Laboratory of Theoretical and Computational Chemistry, Institute of Theoretical \\ Chemistry, Jilin University, Changchun 130023; 2. College of Chemistry, Jilin University, \\ Changchun 130023.
}

Index

Table S1. The selected optimized bond lengths, angles and dihedral angles of (PTZ) and $(\text { FPTZ })_{n}(n=1 \sim 4)$ (bond length : $\AA$, angle: ${ }^{\circ}$ ) obtained by DFT//B3LYP/6-31G ${ }^{*}$ calculations.

page $\mathrm{S} 2$

Figure S1. The optimized structures of $(\mathrm{PTZ})_{\mathrm{n}}$ and $(\mathrm{FPTZ})_{\mathrm{n}} \quad(\mathrm{n}=1 \sim 4)$ by B3LYP/6-31G ${ }^{*}$ from the top (right) and side (left) views. page S3

Figure S2. The electron density isocontours of HOMO and LUMO of the oligomers in $(\mathrm{PTZ})_{\mathrm{n}}$ and $(\mathrm{FPTZ})_{\mathrm{n}}(\mathrm{n}=1 \sim 4)$ by B3LYP/6-31G ${ }^{*}$.

page S5

Table S2. Atom coordinates and total energies (in Hartrees) of optimized computed structure of PTZ, according to B3LYP/6-31G ${ }^{*}$ calculations.

page S6

Table S3. Atom coordinates and total energies (in Hartrees) of optimized computed structure of $(\mathrm{PTZ})_{2}$, according to B3LYP/6-31G ${ }^{*}$ calculations.

page S6

Table S4. Atom coordinates and total energies (in Hartrees) of optimized computed structure of $(\mathrm{PTZ})_{3}$, according to B3LYP/6-31G ${ }^{*}$ calculations. page S7

Table S5. Atom coordinates and total energies (in Hartrees) of optimized computed structure of $(\mathrm{PTZ})_{4}$, according to B3LYP/6-31G ${ }^{*}$ calculations. page S9

Table S6. Atom coordinates and total energies (in Hartrees) of optimized computed structure of FPTZ, according to B3LYP/6-31G ${ }^{*}$ calculations. page S12

Table S7. Atom coordinates and total energies (in Hartrees) of optimized computed structure of $(\mathrm{PFTZ})_{2}$, according to $\mathrm{B} 3 \mathrm{LYP} / 6-31 \mathrm{G}^{*}$ calculations. page S13

Table S8. Atom coordinates and total energies (in Hartrees) of optimized computed structure of $(\mathrm{FPTZ})_{3}$, according to $\mathrm{B} 3 \mathrm{LYP} / 6-31 \mathrm{G}^{*}$ calculations. page $\mathrm{S} 15$

Table S9. Atom coordinates and total energies (in Hartrees) of optimized computed structure of $(\mathrm{FPTZ}) 4$, according to B3LYP/6-31G ${ }^{*}$ calculations.

page S19 
Table S1. The selected optimized bond lengths, angles and dihedral angles of $(\mathrm{PTZ})_{\mathrm{n}}$ and $(\text { FPTZ })_{\mathrm{n}}(\mathrm{n}=1 \sim 4)$ (bond length : $\AA$, angle: ${ }^{\circ}$ ) obtained by DFT//B3LYP/6-31G ${ }^{*}$ calculations.

\begin{tabular}{|c|c|c|c|c|c|c|c|c|}
\hline \multirow[b]{2}{*}{$(\mathrm{n})$} & \multicolumn{3}{|c|}{$(\mathrm{PTZ})_{\mathrm{n}}$} & \multicolumn{5}{|c|}{$(\mathrm{FPTZ})_{\mathrm{n}}$} \\
\hline & (1) & (2) & (3) & (4) & (1) & (2) & (3) & (4) \\
\hline $\mathrm{r}(1,2)$ & 1.840 & 1.840 & 1.840 & 1.841 & 1.840 & 1.840 & 1.841 & 1.840 \\
\hline $\mathrm{r}(3,4)$ & 1.409 & 1.408 & 1.409 & 1.409 & 1.409 & 1.409 & 1.408 & 1.409 \\
\hline $\mathrm{r}(4,5)$ & 1.419 & 1.417 & 1.417 & 1.417 & 1.417 & 1.417 & 1.418 & 1.417 \\
\hline $\mathrm{r}(3,7)$ & 1.394 & 1.391 & 1.391 & 1.391 & 1.391 & 1.391 & 1.391 & 1.391 \\
\hline $\mathrm{r}(8,11)$ & & 1.482 & 1.482 & 1.482 & 1.483 & 1.483 & 1.483 & 1.483 \\
\hline $\mathrm{r}(14,17)$ & & & & & 1.470 & 1.469 & 1.469 & 1.468 \\
\hline $\mathrm{r}(17,18)$ & & & & & 1.415 & 1.415 & 1.415 & 1.415 \\
\hline $\mathrm{r}(15,19)$ & & & & & 1.535 & 1.535 & 1.535 & 1.535 \\
\hline$\theta(1,2,3)$ & 97.6 & 97.6 & 97.6 & 97.6 & 97.6 & 97.8 & 97.6 & 97.8 \\
\hline$\theta(2,3,4)$ & 119.2 & 119.1 & 119.1 & 119.2 & 119.1 & 119.2 & 119.2 & 119.3 \\
\hline$\theta(3,7,8)$ & 120.2 & 121.2 & 121.2 & 121.2 & 121.2 & 121.3 & 121.3 & 121.2 \\
\hline$\theta(7,8,11)$ & & & & & 121.2 & 121.1 & 121.1 & 121.1 \\
\hline$\theta(14,15,19)$ & & & & & 110.8 & 110.8 & 110.8 & 110.8 \\
\hline$\theta(15,19,18)$ & & & & & 101.1 & 101.1 & 101.1 & 101.1 \\
\hline$\theta(15,14,17)$ & & & & & 108.7 & 108.7 & 108.7 & 108.6 \\
\hline$\Phi(1,2,3,4)$ & 35.9 & 36.0 & 36.2 & 35.9 & 36.1 & 36.0 & 35.9 & 35.8 \\
\hline$\Phi(3,4,5,6)$ & 40.2 & 39.5 & 38.9 & 39.3 & 39.2 & 39.3 & 38.9 & 38.8 \\
\hline$\Phi(3,7,8,9)$ & -1.3 & -1.2 & -1.0 & -1.1 & 1.3 & 1.3 & 1.2 & 1.2 \\
\hline$\Phi(7,8,11,12)$ & & 35.8 & 35.5 & 35.1 & 36.7 & 36.4 & 36.5 & 36.5 \\
\hline$\Phi(17,14,15,19)$ & & & & & 0.3 & 0.3 & 0.3 & 0.4 \\
\hline$\Phi(15,14,17,18)$ & & & & & -0.2 & -0.4 & 0.3 & 0.5 \\
\hline
\end{tabular}




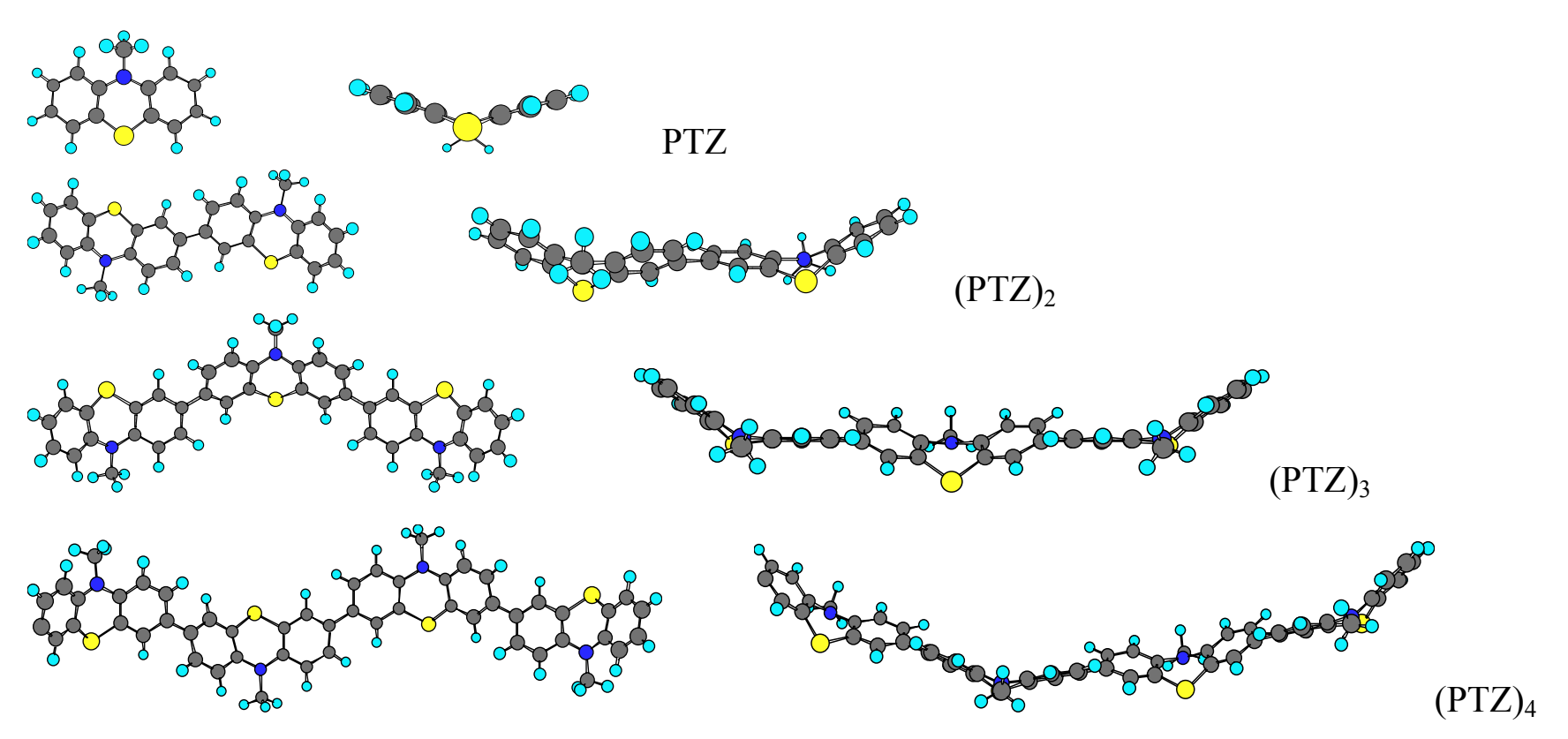




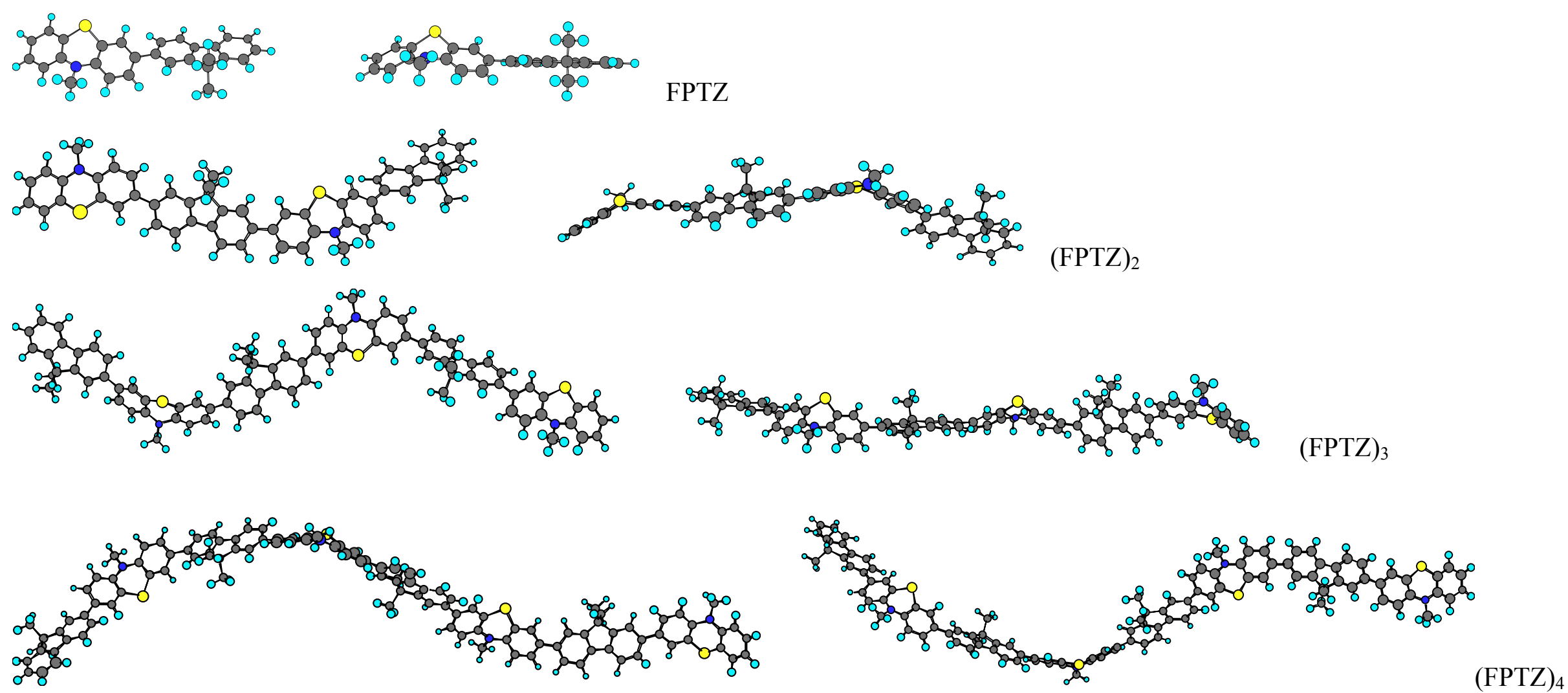

Figure S1. The optimized structures of $(\mathrm{PTZ})_{\mathrm{n}}$ and $(\mathrm{FPTZ})_{\mathrm{n}}(\mathrm{n}=1 \sim 4)$ by B3LYP/6-31G ${ }^{*}$ from the top (right) and side (left) views. 


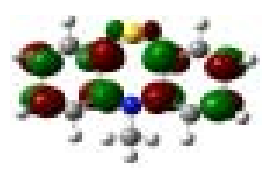

LUMO

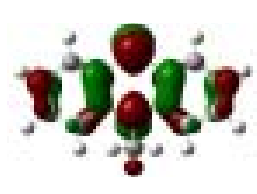

$(\mathrm{PTZ})_{2}$
HOMO

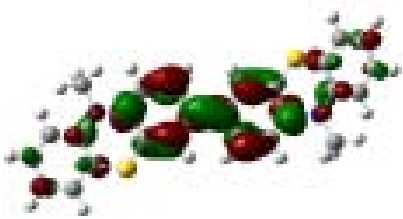

LUMO

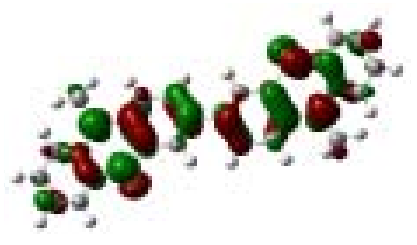

FPTZ
HOMO

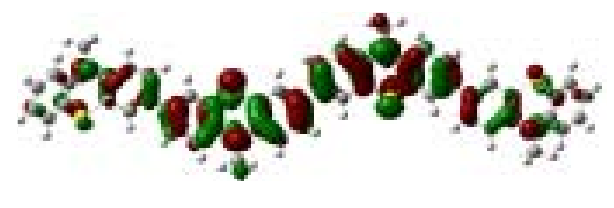

$(\mathrm{PTZ})_{3}$
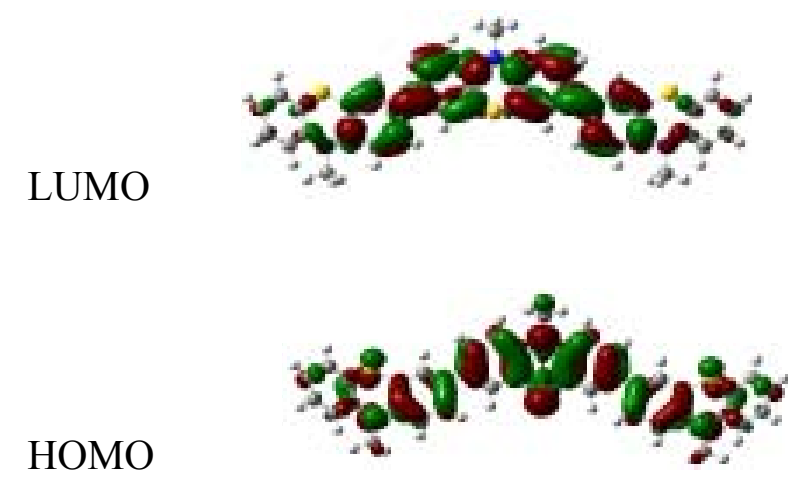

(FPTZ) 4

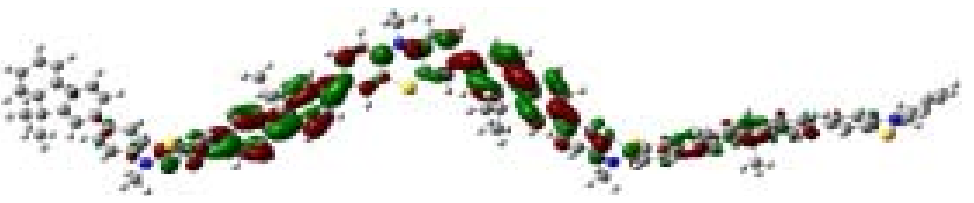

LUMO

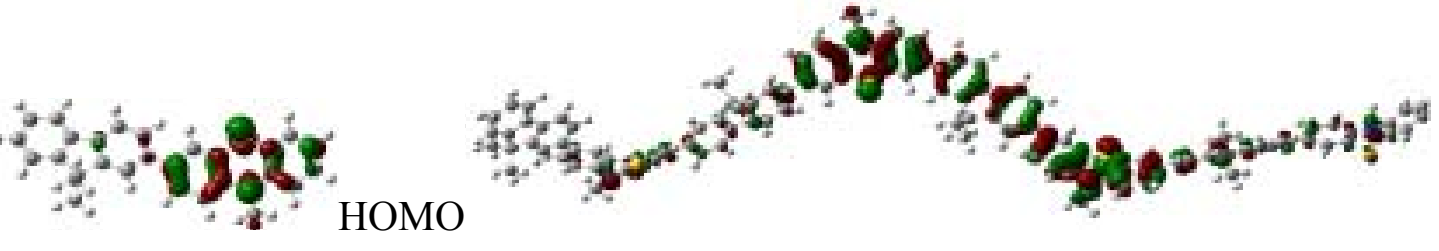

$(\mathrm{FPTZ})_{2}$

$(\mathrm{FPTZ})_{3}$

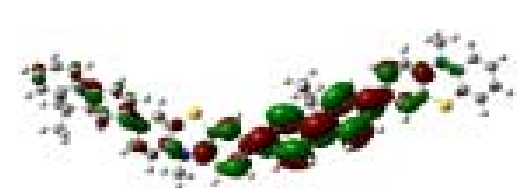

LUMO

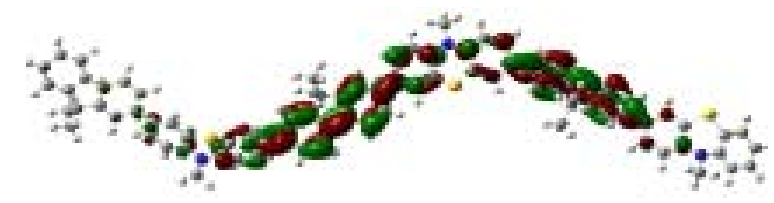

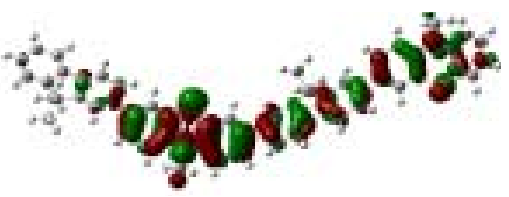

HOMO

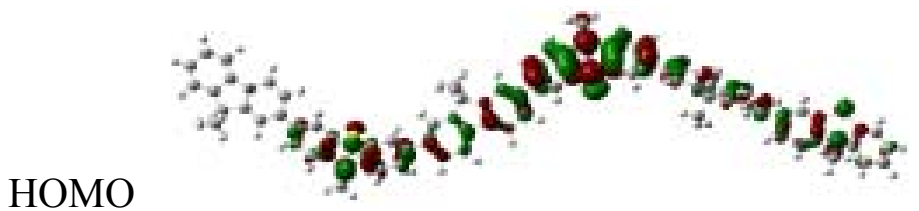

Figure S2. The electron density isocontours of HOMO and LUMO of the oligomers in $(\text { PTZ })_{\mathrm{n}}$ and $(\text { FPTZ })_{\mathrm{n}}(\mathrm{n}=1 \sim 4)$ by B3LYP/6-31G* . 
Table S2. Atom coordinates and total energies (in Hartrees) of optimized computed structure of PTZ, according to B3LYP/6-31G ${ }^{*}$ calculations.

$\begin{array}{rrrrrc}1 & 6 & 0 & -3.528954 & 0.719313 & -0.640189 \\ 2 & 6 & 0 & -2.324231 & 1.335651 & -0.295424 \\ 3 & 6 & 0 & -1.220348 & 0.573352 & 0.115677 \\ 4 & 6 & 0 & -1.350101 & -0.829839 & 0.139668 \\ 5 & 6 & 0 & -2.543833 & -1.440212 & -0.242901 \\ 6 & 6 & 0 & -3.645340 & -0.668375 & -0.617786 \\ 7 & 6 & 0 & 1.350103 & -0.829838 & 0.139669 \\ 8 & 6 & 0 & 1.220348 & 0.573353 & 0.115678 \\ 9 & 6 & 0 & 2.324231 & 1.335653 & -0.295425 \\ 10 & 1 & 0 & 2.247600 & 2.415662 & -0.352289 \\ 11 & 6 & 0 & 3.528954 & 0.719314 & -0.640190 \\ 12 & 6 & 0 & 3.645341 & -0.668373 & -0.617786 \\ 13 & 6 & 0 & 2.543834 & -1.440210 & -0.242899 \\ 14 & 1 & 0 & -4.371842 & 1.335201 & -0.941441 \\ 15 & 1 & 0 & -2.247600 & 2.415661 & -0.352286 \\ 16 & 1 & 0 & -2.610106 & -2.524556 & -0.229376 \\ 17 & 1 & 0 & -4.578283 & -1.150781 & -0.893790 \\ 18 & 1 & 0 & 4.371842 & 1.335203 & -0.941443 \\ 19 & 1 & 0 & 4.578284 & -1.150779 & -0.893789 \\ 20 & 1 & 0 & 2.610107 & -2.524554 & -0.229375 \\ 21 & 16 & 0 & 0.000001 & -1.817102 & 0.755702 \\ 22 & 7 & 0 & -0.000001 & 1.171664 & 0.508547 \\ 23 & 6 & 0 & -0.000003 & 2.592077 & 0.818894 \\ 24 & 1 & 0 & 0.884882 & 2.824522 & 1.416247 \\ 25 & 1 & 0 & -0.000001 & 3.240697 & -0.071685 \\ 26 & 1 & 0 & -0.884894 & 2.824520 & 1.416241\end{array}$

$\mathrm{HF}=-954.9483478$

Table S3. Atom coordinates and total energies (in Hartrees) of optimized computed structure of $(\mathrm{PTZ})_{2}$, according to $\mathrm{B} 3 \mathrm{LYP} / 6-31 \mathrm{G}^{*}$ calculations.

$\begin{array}{rrrrrr}1 & 6 & 0 & -7.897146 & 0.058104 & 1.319845 \\ 2 & 6 & 0 & -6.916052 & -0.867908 & 0.959576 \\ 3 & 6 & 0 & -5.793442 & -0.471661 & 0.216913 \\ 4 & 6 & 0 & -5.667149 & 0.889283 & -0.126930 \\ 5 & 6 & 0 & -6.631458 & 1.814924 & 0.269492 \\ 6 & 6 & 0 & -7.760389 & 1.401696 & 0.979727 \\ 7 & 6 & 0 & -3.065721 & 0.289312 & -0.533168 \\ 8 & 6 & 0 & -3.443795 & -1.014529 & -0.156360 \\ 9 & 6 & 0 & -2.429433 & -1.898955 & 0.239231 \\ 10 & 1 & 0 & -2.679658 & -2.899734 & 0.573157 \\ 11 & 6 & 0 & -1.091851 & -1.507784 & 0.230378\end{array}$




\begin{tabular}{|c|c|c|c|c|c|}
\hline 12 & 6 & 0 & -0.710171 & -0.211029 & -0.140388 \\
\hline 13 & 6 & 0 & -1.731505 & 0.682604 & -0.506683 \\
\hline 14 & 1 & 0 & -8.762654 & -0.279350 & 1.883332 \\
\hline 15 & 1 & 0 & -7.027513 & -1.902369 & 1.264539 \\
\hline 16 & 1 & 0 & -6.500186 & 2.860158 & 0.003823 \\
\hline 17 & 1 & 0 & -8.517239 & 2.125760 & 1.266398 \\
\hline 18 & 1 & 0 & -0.337656 & -2.216473 & 0.559924 \\
\hline 19 & 1 & 0 & -1.479226 & 1.691773 & -0.819017 \\
\hline 20 & 16 & 0 & -4.307617 & 1.406000 & -1.157724 \\
\hline 21 & 7 & 0 & -4.803550 & -1.394898 & -0.193629 \\
\hline 22 & 6 & 0 & -5.122444 & -2.812905 & -0.148933 \\
\hline 23 & 1 & 0 & -4.429889 & -3.352315 & -0.799444 \\
\hline 24 & 1 & 0 & -5.063477 & -3.248513 & 0.861145 \\
\hline 25 & 1 & 0 & -6.135274 & -2.960945 & -0.531167 \\
\hline 26 & 6 & 0 & 0.710172 & 0.211021 & -0.140386 \\
\hline 27 & 6 & 0 & 1.091850 & 1.507776 & 0.230382 \\
\hline 28 & 6 & 0 & 1.731508 & -0.682608 & -0.506685 \\
\hline 29 & 6 & 0 & 2.429431 & 1.898950 & 0.239235 \\
\hline 30 & 1 & 0 & 0.337653 & 2.216464 & 0.559927 \\
\hline 31 & 6 & 0 & 3.065723 & -0.289314 & -0.533169 \\
\hline 32 & 1 & 0 & 1.479231 & -1.691776 & -0.819023 \\
\hline 33 & 6 & 0 & 3.443794 & 1.014526 & -0.156357 \\
\hline 34 & 1 & 0 & 2.679654 & 2.899730 & 0.573163 \\
\hline 35 & 6 & 0 & 5.667152 & -0.889280 & -0.126932 \\
\hline 36 & 6 & 0 & 5.793442 & 0.471664 & 0.216915 \\
\hline 37 & 6 & 0 & 6.631461 & -1.814921 & 0.269491 \\
\hline 38 & 6 & 0 & 6.916049 & 0.867909 & 0.959582 \\
\hline 39 & 6 & 0 & 7.760389 & -1.401694 & 0.979729 \\
\hline 40 & 1 & 0 & 6.500190 & -2.860154 & 0.003818 \\
\hline 41 & 1 & 0 & 7.027508 & 1.902369 & 1.264550 \\
\hline 42 & 6 & 0 & 7.897144 & -0.058103 & 1.319851 \\
\hline 43 & 1 & 0 & 8.517239 & -2.125757 & 1.266400 \\
\hline 44 & 1 & 0 & 8.762650 & 0.279351 & 1.883342 \\
\hline 45 & 16 & 0 & 4.307622 & -1.405996 & -1.157729 \\
\hline 46 & 7 & 0 & 4.803548 & 1.394899 & -0.193625 \\
\hline 47 & 6 & 0 & 5.122437 & 2.812908 & -0.148925 \\
\hline 48 & 1 & 0 & 4.429875 & 3.352318 & -0.799429 \\
\hline 49 & 1 & 0 & 5.063474 & 3.248511 & 0.861156 \\
\hline 50 & 1 & 0 & 6.135264 & 2.960954 & -0.531164 \\
\hline
\end{tabular}

$H F=-1908.7058849$

Table S4. Atom coordinates and total energies (in Hartrees) of optimized computed structure of $(\mathrm{PTZ})_{3}$, according to B3LYP/6-31G ${ }^{*}$ calculations. 


\begin{tabular}{|c|c|c|c|c|c|}
\hline 2 & 6 & 0 & 2.462200 & 0.991316 & 10.591175 \\
\hline 3 & 6 & 0 & 1.565496 & 0.353436 & 9.720567 \\
\hline 4 & 6 & 0 & 0.184273 & 0.509515 & 9.953209 \\
\hline 5 & 6 & 0 & -0.274318 & 1.284769 & 11.017394 \\
\hline 6 & 6 & 0 & 0.628858 & 1.887069 & 11.895557 \\
\hline 7 & 6 & 0 & -0.085312 & -0.279690 & 7.386086 \\
\hline 8 & 6 & 0 & 1.320850 & -0.366975 & 7.402615 \\
\hline 9 & 6 & 0 & 1.987560 & -0.389842 & 6.168729 \\
\hline 10 & 1 & 0 & 3.070921 & -0.419346 & 6.136308 \\
\hline 11 & 6 & 0 & 1.279104 & -0.353872 & 4.969116 \\
\hline 12 & 6 & 0 & -0.119411 & -0.260579 & 4.946482 \\
\hline 13 & 6 & 0 & -0.783137 & -0.210069 & 6.184594 \\
\hline 14 & 1 & 0 & 2.713138 & 2.206716 & 12.343159 \\
\hline 15 & 1 & 0 & 3.530474 & 0.914744 & 10.422427 \\
\hline 16 & 1 & 0 & -1.344668 & 1.400440 & 11.163265 \\
\hline 17 & 1 & 0 & 0.263482 & 2.471934 & 12.734429 \\
\hline 18 & 1 & 0 & 1.832968 & -0.354308 & 4.034857 \\
\hline 19 & 1 & 0 & -1.867555 & -0.157680 & 6.213860 \\
\hline 20 & 16 & 0 & -0.977134 & -0.371639 & 8.927314 \\
\hline 21 & 7 & 0 & 2.009968 & -0.435688 & 8.633692 \\
\hline 22 & 6 & 0 & 3.396829 & -0.872782 & 8.621834 \\
\hline 23 & 1 & 0 & 3.494293 & -1.725166 & 7.945240 \\
\hline 24 & 1 & 0 & 4.104442 & -0.091216 & 8.302633 \\
\hline 25 & 1 & 0 & 3.673602 & -1.201056 & 9.626507 \\
\hline 26 & 6 & 0 & -0.874244 & -0.203609 & 3.672690 \\
\hline 27 & 6 & 0 & -2.028821 & 0.579926 & 3.538312 \\
\hline 28 & 6 & 0 & -0.450740 & -0.927417 & 2.544558 \\
\hline 29 & 6 & 0 & -2.729093 & 0.644713 & 2.335313 \\
\hline 30 & 1 & 0 & -2.366798 & 1.187150 & 4.372932 \\
\hline 31 & 6 & 0 & -1.163556 & -0.894719 & 1.350097 \\
\hline 32 & 1 & 0 & 0.427914 & -1.562525 & 2.609765 \\
\hline 33 & 6 & 0 & -2.320671 & -0.101304 & 1.219911 \\
\hline 34 & 1 & 0 & -3.592205 & 1.297391 & 2.266796 \\
\hline 35 & 6 & 0 & -1.163556 & -0.894719 & -1.350097 \\
\hline 36 & 6 & 0 & -2.320671 & -0.101304 & -1.219911 \\
\hline 37 & 6 & 0 & -0.450740 & -0.927417 & -2.544558 \\
\hline 38 & 6 & 0 & -2.729093 & 0.644713 & -2.335313 \\
\hline 39 & 6 & 0 & -0.874244 & -0.203609 & -3.672690 \\
\hline 40 & 1 & 0 & 0.427914 & -1.562525 & -2.609765 \\
\hline 41 & 1 & 0 & -3.592205 & 1.297391 & -2.266796 \\
\hline 42 & 6 & 0 & -2.028821 & 0.579926 & -3.538312 \\
\hline 43 & 1 & 0 & -2.366798 & 1.187150 & -4.372932 \\
\hline 44 & 16 & 0 & -0.678336 & -1.953882 & 0.000000 \\
\hline 45 & 7 & 0 & -3.031890 & -0.080654 & 0.000000 \\
\hline
\end{tabular}




\begin{tabular}{|c|c|c|c|c|c|}
\hline 46 & 6 & 0 & -4.386925 & 0.447402 & 0.000000 \\
\hline 47 & 1 & 0 & -4.911115 & 0.079021 & 0.884971 \\
\hline 48 & 1 & 0 & -4.432161 & 1.547934 & 0.000000 \\
\hline 49 & 1 & 0 & -4.911115 & 0.079021 & -0.884971 \\
\hline 50 & 6 & 0 & -0.119411 & -0.260579 & -4.946482 \\
\hline 51 & 6 & 0 & 1.279104 & -0.353872 & -4.969116 \\
\hline 52 & 6 & 0 & -0.783137 & -0.210069 & -6.184594 \\
\hline 53 & 6 & 0 & 1.987560 & -0.389842 & -6.168729 \\
\hline 54 & 1 & 0 & 1.832968 & -0.354308 & -4.034857 \\
\hline 55 & 6 & 0 & -0.085312 & -0.279690 & -7.386086 \\
\hline 56 & 1 & 0 & -1.867555 & -0.157680 & -6.213860 \\
\hline 57 & 6 & 0 & 1.320850 & -0.366975 & -7.402615 \\
\hline 58 & 1 & 0 & 3.070921 & -0.419346 & -6.136308 \\
\hline 59 & 6 & 0 & 0.184273 & 0.509515 & -9.953209 \\
\hline 60 & 6 & 0 & 1.565496 & 0.353436 & -9.720567 \\
\hline 61 & 6 & 0 & -0.274318 & 1.284769 & -11.017394 \\
\hline 62 & 6 & 0 & 2.462200 & 0.991316 & -10.591175 \\
\hline 63 & 6 & 0 & 0.628858 & 1.887069 & -11.895557 \\
\hline 64 & 1 & 0 & -1.344668 & 1.400440 & -11.163265 \\
\hline 65 & 1 & 0 & 3.530474 & 0.914744 & -10.422427 \\
\hline 66 & 6 & 0 & 1.995905 & 1.734635 & -11.677411 \\
\hline 67 & 1 & 0 & 0.263482 & 2.471934 & -12.734429 \\
\hline 68 & 1 & 0 & 2.713138 & 2.206716 & -12.343159 \\
\hline 69 & 6 & 0 & 3.396829 & -0.872782 & -8.621834 \\
\hline 70 & 1 & 0 & 4.104442 & -0.091216 & -8.302633 \\
\hline 71 & 1 & 0 & 3.494293 & -1.725166 & -7.945240 \\
\hline 72 & 1 & 0 & 3.673602 & -1.201056 & -9.626507 \\
\hline 73 & 7 & 0 & 2.009968 & -0.435688 & -8.633692 \\
\hline 74 & 16 & 0 & -0.977134 & -0.371639 & -8.927314 \\
\hline
\end{tabular}

Table S5. Atom coordinates and total energies (in Hartrees) of optimized computed structure of $(\mathrm{PTZ})_{4}$, according to B3LYP/6-31G ${ }^{*}$ calculations.

$\begin{array}{rlllll}1 & 6 & 0 & 7.606956 & 2.130292 & -0.041812 \\ 2 & 6 & 0 & 6.413663 & 2.748066 & -0.411354 \\ 3 & 6 & 0 & 5.436588 & 2.054889 & -1.140749 \\ 4 & 6 & 0 & 5.692191 & 0.707181 & -1.462698 \\ 5 & 6 & 0 & 6.871909 & 0.088414 & -1.061703 \\ 6 & 6 & 0 & 7.865082 & 0.788471 & -0.354807 \\ 7 & 6 & 0 & 3.029311 & 0.563843 & -1.886686 \\ 8 & 6 & 0 & 3.030941 & 1.924587 & -1.521312 \\ 9 & 6 & 0 & 1.810182 & 2.498190 & -1.136504 \\ 10 & 1 & 0 & 1.773714 & 3.531611 & -0.810333 \\ 11 & 6 & 0 & 0.632512 & 1.753271 & -1.144561\end{array}$




\begin{tabular}{|c|c|c|c|c|c|}
\hline 12 & 6 & 0 & 0.624443 & 0.398710 & -1.505680 \\
\hline 13 & 6 & 0 & 1.854606 & -0.181104 & -1.861697 \\
\hline 14 & 1 & 0 & 8.329762 & 2.695592 & 0.539137 \\
\hline 15 & 1 & 0 & 6.241292 & 3.775442 & -0.110613 \\
\hline 16 & 1 & 0 & 7.038254 & -0.947334 & -1.342984 \\
\hline 17 & 1 & 0 & -0.288698 & 2.229278 & -0.821670 \\
\hline 18 & 1 & 0 & 1.892162 & -1.222735 & -2.166851 \\
\hline 19 & 16 & 0 & 4.534695 & -0.175343 & -2.493330 \\
\hline 20 & 7 & 0 & 4.232861 & 2.665296 & -1.556891 \\
\hline 21 & 6 & 0 & 4.149630 & 4.117001 & -1.527717 \\
\hline 22 & 1 & 0 & 3.337681 & 4.438864 & -2.184087 \\
\hline 23 & 1 & 0 & 3.970229 & 4.529258 & -0.522174 \\
\hline 24 & 1 & 0 & 5.083946 & 4.533503 & -1.911475 \\
\hline 25 & 6 & 0 & -0.624445 & -0.398649 & -1.505699 \\
\hline 26 & 6 & 0 & -0.632516 & -1.753226 & -1.144639 \\
\hline 27 & 6 & 0 & -1.854608 & 0.181180 & -1.861694 \\
\hline 28 & 6 & 0 & -1.810185 & -2.498145 & -1.136617 \\
\hline 29 & 1 & 0 & 0.288694 & -2.229247 & -0.821767 \\
\hline 30 & 6 & 0 & -3.029312 & -0.563765 & -1.886717 \\
\hline 31 & 1 & 0 & -1.892162 & 1.222825 & -2.166802 \\
\hline 32 & 6 & 0 & -3.030943 & -1.924525 & -1.521402 \\
\hline 33 & 1 & 0 & -1.773718 & -3.531580 & -0.810491 \\
\hline 34 & 6 & 0 & -5.692193 & -0.707120 & -1.462739 \\
\hline 35 & 6 & 0 & -5.436590 & -2.054842 & -1.140847 \\
\hline 36 & 6 & 0 & -6.871911 & -0.088370 & -1.061718 \\
\hline 37 & 6 & 0 & -6.413666 & -2.748049 & -0.411482 \\
\hline 38 & 6 & 0 & -7.865084 & -0.788455 & -0.354851 \\
\hline 39 & 1 & 0 & -7.038254 & 0.947390 & -1.342955 \\
\hline 40 & 1 & 0 & -6.241296 & -3.775438 & -0.110784 \\
\hline 41 & 6 & 0 & -7.606958 & -2.130290 & -0.041913 \\
\hline 42 & 1 & 0 & -8.329765 & -2.695614 & 0.539011 \\
\hline 43 & 16 & 0 & -4.534695 & 0.175447 & -2.493332 \\
\hline 44 & 7 & 0 & -4.232863 & -2.665232 & -1.557014 \\
\hline 45 & 6 & 0 & -4.149633 & -4.116938 & -1.527902 \\
\hline 46 & 1 & 0 & -3.337685 & -4.438774 & -2.184286 \\
\hline 47 & 1 & 0 & -3.970231 & -4.529237 & -0.522376 \\
\hline 48 & 1 & 0 & -5.083950 & -4.533423 & -1.911676 \\
\hline 49 & 6 & 0 & -9.126569 & -0.124448 & 0.049202 \\
\hline 50 & 6 & 0 & -9.156773 & 1.223824 & 0.431840 \\
\hline 51 & 6 & 0 & -10.342416 & -0.829644 & 0.073009 \\
\hline 52 & 6 & 0 & -10.342077 & 1.843701 & 0.822565 \\
\hline 53 & 1 & 0 & -8.232668 & 1.793153 & 0.468474 \\
\hline 54 & 6 & 0 & -11.535266 & -0.209002 & 0.430243 \\
\hline 55 & 1 & 0 & -10.368243 & -1.871175 & -0.233785 \\
\hline
\end{tabular}




\begin{tabular}{|c|c|c|c|c|c|}
\hline 56 & 6 & 0 & -11.558782 & 1.145843 & 0.815850 \\
\hline 57 & 1 & 0 & -10.309827 & 2.876804 & 1.150030 \\
\hline 58 & 6 & 0 & -13.938243 & -0.336918 & 1.655994 \\
\hline 59 & 6 & 0 & -13.727683 & 1.029959 & 1.927487 \\
\hline 60 & 6 & 0 & -14.865673 & -1.073091 & 2.392153 \\
\hline 61 & 6 & 0 & -14.479917 & 1.628212 & 2.949672 \\
\hline 62 & 6 & 0 & -15.631058 & -0.457570 & 3.384608 \\
\hline 63 & 1 & 0 & -14.996319 & -2.128828 & 2.171559 \\
\hline 64 & 1 & 0 & -14.320766 & 2.670537 & 3.202219 \\
\hline 65 & 6 & 0 & -15.434310 & 0.894031 & 3.656815 \\
\hline 66 & 1 & 0 & -16.366242 & -1.033144 & 3.939121 \\
\hline 67 & 1 & 0 & -16.012238 & 1.386264 & 4.434125 \\
\hline 68 & 6 & 0 & -12.834689 & 3.211336 & 1.212877 \\
\hline 69 & 1 & 0 & -12.390720 & 3.644572 & 2.123180 \\
\hline 70 & 1 & 0 & -12.304063 & 3.610895 & 0.345378 \\
\hline 71 & 1 & 0 & -13.878045 & 3.529632 & 1.151010 \\
\hline 72 & 7 & 0 & -12.780891 & 1.758767 & 1.170376 \\
\hline 73 & 16 & 0 & -13.074884 & -1.098227 & 0.294581 \\
\hline 74 & 6 & 0 & 9.126568 & 0.124447 & 0.049217 \\
\hline 75 & 6 & 0 & 9.156773 & -1.223842 & 0.431796 \\
\hline 76 & 6 & 0 & 10.342415 & 0.829643 & 0.073053 \\
\hline 77 & 6 & 0 & 10.342078 & -1.843735 & 0.822493 \\
\hline 78 & 1 & 0 & 8.232668 & -1.793173 & 0.468407 \\
\hline 79 & 6 & 0 & 11.535266 & 0.208986 & 0.430257 \\
\hline 80 & 1 & 0 & 10.368241 & 1.871187 & -0.233696 \\
\hline 81 & 6 & 0 & 11.558783 & -1.145877 & 0.815806 \\
\hline 82 & 1 & 0 & 10.309830 & -2.876852 & 1.149913 \\
\hline 83 & 6 & 0 & 13.938245 & 0.336850 & 1.656009 \\
\hline 84 & 6 & 0 & 13.727686 & -1.030039 & 1.927444 \\
\hline 85 & 6 & 0 & 14.865676 & 1.072991 & 2.392197 \\
\hline 86 & 6 & 0 & 14.479923 & -1.628336 & 2.949601 \\
\hline 87 & 6 & 0 & 15.631065 & 0.457428 & 3.384623 \\
\hline 88 & 1 & 0 & 14.996321 & 2.128738 & 2.171648 \\
\hline 89 & 1 & 0 & 14.320775 & -2.670672 & 3.202103 \\
\hline 90 & 6 & 0 & 15.434318 & -0.894185 & 3.656773 \\
\hline 91 & 1 & 0 & 16.366250 & 1.032979 & 3.939159 \\
\hline 92 & 1 & 0 & 16.012249 & -1.386450 & 4.434060 \\
\hline 93 & 16 & 0 & 13.074883 & 1.098217 & 0.294631 \\
\hline 94 & 7 & 0 & 12.780893 & -1.758815 & 1.170304 \\
\hline 95 & 6 & 0 & 12.834692 & -3.211386 & 1.212742 \\
\hline 96 & 1 & 0 & 12.390726 & -3.644661 & 2.123027 \\
\hline 97 & 1 & 0 & 12.304064 & -3.610908 & 0.345228 \\
\hline 98 & 1 & 0 & 13.878048 & -3.529678 & 1.150858 \\
\hline
\end{tabular}

$\mathrm{HF}=-3816.2210058$ 
Table S6. Atom coordinates and total energies (in Hartrees) of optimized computed structure of FPTZ, according to B3LYP/6-31G ${ }^{*}$ calculations.

\begin{tabular}{|c|c|c|c|c|c|}
\hline 1 & 6 & 0 & 0.854245 & 1.467436 & -0.541073 \\
\hline 2 & 6 & 0 & 2.199824 & 1.826764 & -0.492150 \\
\hline 3 & 6 & 0 & 3.158919 & 0.961408 & 0.054573 \\
\hline 4 & 6 & 0 & 2.718786 & -0.292409 & 0.523173 \\
\hline 5 & 6 & 0 & 1.379324 & -0.658215 & 0.436474 \\
\hline 6 & 6 & 0 & 0.410816 & 0.219388 & -0.081560 \\
\hline 7 & 6 & 0 & 5.323883 & -0.985210 & 0.362830 \\
\hline 8 & 6 & 0 & 5.511864 & 0.333070 & -0.098645 \\
\hline 9 & 6 & 0 & 6.692614 & 0.631192 & -0.795558 \\
\hline 10 & 1 & 0 & 6.853927 & 1.628381 & -1.189480 \\
\hline 11 & 6 & 0 & 7.669168 & -0.346506 & -0.996528 \\
\hline 12 & 6 & 0 & 7.471997 & -1.647411 & -0.540084 \\
\hline 13 & 6 & 0 & 6.286367 & -1.965571 & 0.125247 \\
\hline 14 & 1 & 0 & 0.144138 & 2.157883 & -0.986703 \\
\hline 15 & 1 & 0 & 2.501195 & 2.785233 & -0.899811 \\
\hline 16 & 1 & 0 & 1.078025 & -1.628445 & 0.820364 \\
\hline 17 & 1 & 0 & 8.579931 & -0.084218 & -1.527766 \\
\hline 18 & 1 & 0 & 8.225956 & -2.411889 & -0.702558 \\
\hline 19 & 1 & 0 & 6.107840 & -2.977081 & 0.479181 \\
\hline 20 & 16 & 0 & 3.882236 & -1.370322 & 1.338086 \\
\hline 21 & 7 & 0 & 4.523129 & 1.313298 & 0.152468 \\
\hline 22 & 6 & 0 & 4.884891 & 2.712855 & -0.004387 \\
\hline 23 & 1 & 0 & 5.874143 & 2.874611 & 0.430287 \\
\hline 24 & 1 & 0 & 4.904602 & 3.050045 & -1.052918 \\
\hline 25 & 1 & 0 & 4.166896 & 3.327753 & 0.543515 \\
\hline 26 & 6 & 0 & -1.019777 & -0.165578 & -0.143334 \\
\hline 27 & 6 & 0 & -1.399960 & -1.488679 & -0.440068 \\
\hline 28 & 6 & 0 & -2.030620 & 0.788150 & 0.092657 \\
\hline 29 & 6 & 0 & -2.740278 & -1.866352 & -0.501842 \\
\hline 30 & 1 & 0 & -0.630350 & -2.225294 & -0.652483 \\
\hline 31 & 6 & 0 & -3.366550 & 0.419726 & 0.029257 \\
\hline 32 & 1 & 0 & -1.751010 & 1.806363 & 0.351670 \\
\hline 33 & 6 & 0 & -3.728895 & -0.908961 & -0.267334 \\
\hline 34 & 1 & 0 & -3.003202 & -2.893894 & -0.740361 \\
\hline 35 & 6 & 0 & -4.606960 & 1.283645 & 0.259848 \\
\hline 36 & 6 & 0 & -5.192609 & -1.007609 & -0.261275 \\
\hline 37 & 6 & 0 & -5.723711 & 0.262044 & 0.044365 \\
\hline 38 & 6 & 0 & -6.038331 & -2.093918 & -0.496265 \\
\hline 39 & 6 & 0 & -7.099858 & 0.446442 & 0.115285 \\
\hline 40 & 6 & 0 & -7.419751 & -1.901686 & -0.423783 \\
\hline 41 & 1 & 0 & -5.632837 & -3.074706 & -0.73202 \\
\hline
\end{tabular}




$\begin{array}{llllll}42 & 6 & 0 & -7.947997 & -0.642438 & -0.120553 \\ 43 & 1 & 0 & -7.520792 & 1.421438 & 0.350747 \\ 44 & 1 & 0 & -8.090155 & -2.737816 & -0.604497 \\ 45 & 1 & 0 & -9.025137 & -0.508032 & -0.067489 \\ 46 & 6 & 0 & -4.691783 & 2.435081 & -0.767028 \\ 47 & 1 & 0 & -3.851189 & 3.127944 & -0.643458 \\ 48 & 1 & 0 & -4.671279 & 2.051723 & -1.792208 \\ 49 & 1 & 0 & -5.618834 & 3.004594 & -0.633113 \\ 50 & 6 & 0 & -4.636624 & 1.855007 & 1.695310 \\ 51 & 1 & 0 & -4.580732 & 1.054045 & 2.439275 \\ 52 & 1 & 0 & -3.792175 & 2.534364 & 1.859832 \\ 53 & 1 & 0 & -5.560612 & 2.419063 & 1.868043\end{array}$

$H F=-1533.8086326$

Table S7. Atom coordinates and total energies (in Hartrees) of optimized computed structure of $(\mathrm{FPTZ})_{2}$, according to B3LYP/6-31G ${ }^{*}$ calculations.

$\begin{array}{cccccc}1 & 6 & 0 & 8.166612 & -1.965767 & -0.382156 \\ 2 & 6 & 0 & 7.197426 & -2.956620 & -0.237498 \\ 3 & 6 & 0 & 5.932952 & -2.659194 & 0.292312 \\ 4 & 6 & 0 & 5.667779 & -1.319885 & 0.640906 \\ 5 & 6 & 0 & 6.628553 & -0.330333 & 0.458947 \\ 6 & 6 & 0 & 7.908064 & -0.631013 & -0.040040 \\ 7 & 6 & 0 & 3.074230 & -2.082461 & 0.587552 \\ 8 & 6 & 0 & 3.590933 & -3.344435 & 0.234407 \\ 9 & 6 & 0 & 2.714848 & -4.265169 & -0.359149 \\ 10 & 1 & 0 & 3.076022 & -5.241223 & -0.663282 \\ 11 & 6 & 0 & 1.371608 & -3.952368 & -0.559679 \\ 12 & 6 & 0 & 0.851734 & -2.697062 & -0.214213 \\ 13 & 6 & 0 & 1.741385 & -1.763657 & 0.346035 \\ 14 & 1 & 0 & 9.128055 & -2.233246 & -0.810655 \\ 15 & 1 & 0 & 7.429497 & -3.966482 & -0.556823 \\ 16 & 1 & 0 & 6.390355 & 0.687455 & 0.753721 \\ 17 & 1 & 0 & 0.715802 & -4.708104 & -0.981674 \\ 18 & 1 & 0 & 1.391530 & -0.765522 & 0.592039 \\ 19 & 16 & 0 & 4.122624 & -0.914878 & 1.433010 \\ 20 & 7 & 0 & 4.946909 & -3.650998 & 0.487288 \\ 21 & 6 & 0 & 5.356253 & -5.046082 & 0.452973 \\ 22 & 1 & 0 & 4.598717 & -5.650558 & 0.957375 \\ 23 & 1 & 0 & 5.497067 & -5.440703 & -0.565663 \\ 24 & 1 & 0.296516 & -5.152919 & 0.998970 \\ 25 & 6 & -8.483042 & 0.511876 & 0.073691 \\ 26 & 6 & -8.583503 & 1.785238 & 0.651755 \\ 27 & 6 & 0 & -9.683177 & -0.158513 & -0.221033 \\ 28 & 6 & -9.820104 & 2.368024 & 0.922118\end{array}$




\begin{tabular}{|c|c|c|c|c|c|}
\hline 29 & 1 & 0 & -7.681367 & 2.354326 & 0.856222 \\
\hline 30 & 6 & 0 & -10.920909 & 0.401307 & 0.079228 \\
\hline 31 & 1 & 0 & -9.652346 & -1.154508 & -0.652986 \\
\hline 32 & 6 & 0 & -11.015099 & 1.683014 & 0.656744 \\
\hline 33 & 1 & 0 & -9.847681 & 3.372627 & 1.329165 \\
\hline 34 & 6 & 0 & -13.511498 & 0.785450 & -0.580089 \\
\hline 35 & 6 & 0 & -13.354422 & 2.033059 & 0.056369 \\
\hline 36 & 6 & 0 & -14.561362 & 0.569473 & -1.471706 \\
\hline 37 & 6 & 0 & -14.284929 & 3.044814 & -0.225367 \\
\hline 38 & 6 & 0 & -15.499213 & 1.574173 & -1.717933 \\
\hline 39 & 1 & 0 & -14.648911 & -0.397141 & -1.959856 \\
\hline 40 & 1 & 0 & -14.175718 & 4.024746 & 0.225746 \\
\hline 41 & 6 & 0 & -15.355915 & 2.808897 & -1.089833 \\
\hline 42 & 1 & 0 & -16.326130 & 1.391013 & -2.397590 \\
\hline 43 & 1 & 0 & -16.069139 & 3.606281 & -1.279556 \\
\hline 44 & 16 & 0 & -12.408406 & -0.551634 & -0.163396 \\
\hline 45 & 7 & 0 & -12.281065 & 2.232541 & 0.956057 \\
\hline 46 & 6 & 0 & -12.339742 & 3.372357 & 1.857082 \\
\hline 47 & 1 & 0 & -11.655215 & 3.200186 & 2.691058 \\
\hline 48 & 1 & 0 & -12.073769 & 4.328542 & 1.379198 \\
\hline 49 & 1 & 0 & -13.352514 & 3.455667 & 2.258518 \\
\hline 50 & 6 & 0 & 8.936641 & 0.425013 & -0.198986 \\
\hline 51 & 6 & 0 & 8.579165 & 1.726489 & -0.601101 \\
\hline 52 & 6 & 0 & 10.296011 & 0.147540 & 0.051070 \\
\hline 53 & 6 & 0 & 9.532931 & 2.731750 & -0.750677 \\
\hline 54 & 1 & 0 & 7.538740 & 1.944029 & -0.825176 \\
\hline 55 & 6 & 0 & 11.250890 & 1.142694 & -0.099265 \\
\hline 56 & 1 & 0 & 10.582508 & -0.845168 & 0.389530 \\
\hline 57 & 6 & 0 & 10.874793 & 2.440088 & -0.499104 \\
\hline 58 & 1 & 0 & 9.227504 & 3.725370 & -1.068997 \\
\hline 59 & 6 & 0 & 12.760764 & 1.059412 & 0.128168 \\
\hline 60 & 6 & 0 & 12.078117 & 3.276294 & -0.567490 \\
\hline 61 & 6 & 0 & 13.189711 & 2.488708 & -0.204681 \\
\hline 62 & 6 & 0 & 12.240922 & 4.619886 & -0.912016 \\
\hline 63 & 6 & 0 & 14.464331 & 3.043601 & -0.186584 \\
\hline 64 & 6 & 0 & 13.524142 & 5.170736 & -0.892340 \\
\hline 65 & 1 & 0 & 11.386228 & 5.230685 & -1.191865 \\
\hline 66 & 6 & 0 & 14.627980 & 4.390444 & -0.532797 \\
\hline 67 & 1 & 0 & 15.329246 & 2.446036 & 0.092504 \\
\hline 68 & 1 & 0 & 13.666319 & 6.214839 & -1.158512 \\
\hline 69 & 1 & 0 & 15.620514 & 4.833000 & -0.521717 \\
\hline 70 & 6 & 0 & 13.418340 & 0.037824 & -0.826658 \\
\hline 71 & 1 & 0 & 14.507716 & 0.039096 & -0.704484 \\
\hline 72 & 1 & 0 & 13.058180 & -0.976163 & -0.616900 \\
\hline
\end{tabular}




\begin{tabular}{|c|c|c|c|c|c|}
\hline 73 & 1 & 0 & 13.191519 & 0.272276 & -1.871607 \\
\hline 74 & 6 & 0 & 13.089529 & 0.698071 & 1.594164 \\
\hline 75 & 1 & 0 & 12.635557 & 1.413284 & 2.287381 \\
\hline 76 & 1 & 0 & 12.713276 & -0.301871 & 1.839372 \\
\hline 77 & 1 & 0 & 14.173056 & 0.699942 & 1.760604 \\
\hline 78 & 6 & 0 & -0.576626 & -2.360798 & -0.428065 \\
\hline 79 & 6 & 0 & -1.270063 & -2.843247 & -1.555146 \\
\hline 80 & 6 & 0 & -1.272242 & -1.553026 & 0.494606 \\
\hline 81 & 6 & 0 & -2.614285 & -2.543841 & -1.768937 \\
\hline 82 & 1 & 0 & -0.735929 & -3.441267 & -2.287973 \\
\hline 83 & 6 & 0 & -2.608912 & -1.247196 & 0.285935 \\
\hline 84 & 1 & 0 & -0.758304 & -1.195855 & 1.383582 \\
\hline 85 & 6 & 0 & -3.288166 & -1.741837 & -0.845414 \\
\hline 86 & 1 & 0 & -3.121258 & -2.924992 & -2.651898 \\
\hline 87 & 6 & 0 & -3.541990 & -0.404835 & 1.156729 \\
\hline 88 & 6 & 0 & -4.673834 & -1.267843 & -0.801616 \\
\hline 89 & 6 & 0 & -4.842522 & -0.481792 & 0.355937 \\
\hline 90 & 6 & 0 & -5.748893 & -1.474114 & -1.668605 \\
\hline 91 & 6 & 0 & -6.071773 & 0.092434 & 0.644051 \\
\hline 92 & 6 & 0 & -6.980261 & -0.891311 & -1.374536 \\
\hline 93 & 1 & 0 & -5.632781 & -2.071358 & -2.569529 \\
\hline 94 & 6 & 0 & -7.166780 & -0.103012 & -0.222308 \\
\hline 95 & 1 & 0 & -6.209104 & 0.674408 & 1.552116 \\
\hline 96 & 1 & 0 & -7.809197 & -1.024723 & -2.063791 \\
\hline 97 & 6 & 0 & -3.039338 & 1.051294 & 1.275293 \\
\hline 98 & 1 & 0 & -3.750956 & 1.661091 & 1.844062 \\
\hline 99 & 1 & 0 & -2.075297 & 1.086961 & 1.795935 \\
\hline 100 & 1 & 0 & -2.912110 & 1.505748 & 0.287582 \\
\hline 101 & 6 & 0 & -3.708884 & -1.021497 & 2.563740 \\
\hline 102 & 1 & 0 & -4.066371 & -2.054258 & 2.501759 \\
\hline 103 & 1 & 0 & -2.754086 & -1.021341 & 3.102133 \\
\hline 104 & 1 & 0 & -4.429078 & -0.445142 & 3.155981 \\
\hline
\end{tabular}

$\mathrm{HF}=-3066.4264012$

Table S8. Atom coordinates and total energies (in Hartrees) of optimized computed structure of (FPTZ) 3 , according to B3LYP/6-31G ${ }^{*}$ calculations.

$\begin{array}{cccccc}1 & 6 & 0 & 0.036848 & 3.280344 & -1.038981 \\ 2 & 6 & 0 & 1.092037 & 4.188339 & -0.973992 \\ 3 & 6 & 0 & 2.296992 & 3.851610 & -0.339345 \\ 4 & 6 & 0 & 2.411434 & 2.556673 & 0.204419 \\ 5 & 6 & 0 & 1.366781 & 1.643594 & 0.101901 \\ 6 & 6 & 0 & 0.147774 & 1.987659 & -0.508031 \\ 7 & 6 & 0 & 5.066941 & 3.050621 & 0.181163 \\ 8 & 6 & 0 & 4.696074 & 4.291828 & -0.372152\end{array}$




\begin{tabular}{|c|c|c|c|c|c|}
\hline 9 & 6 & 0 & 5.681354 & 5.024450 & -1.050420 \\
\hline 10 & 1 & 0 & 5.433706 & 5.976557 & -1.506216 \\
\hline 11 & 6 & 0 & 6.989070 & 4.552202 & -1.146062 \\
\hline 12 & 6 & 0 & 7.363116 & 3.314770 & -0.603602 \\
\hline 13 & 6 & 0 & 6.365457 & 2.568378 & 0.047360 \\
\hline 14 & 1 & 0 & -0.873832 & 3.572141 & -1.553824 \\
\hline 15 & 1 & 0 & 0.974888 & 5.160855 & -1.438912 \\
\hline 16 & 1 & 0 & 1.489659 & 0.658829 & 0.543147 \\
\hline 17 & 1 & 0 & 7.732769 & 5.167118 & -1.644200 \\
\hline 18 & 1 & 0 & 6.601406 & 1.587050 & 0.447989 \\
\hline 19 & 16 & 0 & 3.873358 & 2.127942 & 1.130644 \\
\hline 20 & 7 & 0 & 3.370917 & 4.762492 & -0.230361 \\
\hline 21 & 6 & 0 & 3.109186 & 6.170114 & -0.485586 \\
\hline 22 & 1 & 0 & 3.906348 & 6.766440 & -0.035498 \\
\hline 23 & 1 & 0 & 3.047163 & 6.421084 & -1.556293 \\
\hline 24 & 1 & 0 & 2.165803 & 6.447279 & -0.009306 \\
\hline 25 & 6 & 0 & 16.267617 & -0.833859 & 0.417678 \\
\hline 26 & 6 & 0 & 16.214036 & -2.008306 & 1.181496 \\
\hline 27 & 6 & 0 & 17.539861 & -0.363387 & 0.047897 \\
\hline 28 & 6 & 0 & 17.371267 & -2.688515 & 1.556066 \\
\hline 29 & 1 & 0 & 15.249853 & -2.427987 & 1.452938 \\
\hline 30 & 6 & 0 & 18.700929 & -1.016645 & 0.448441 \\
\hline 31 & 1 & 0 & 17.629678 & 0.553007 & -0.527969 \\
\hline 32 & 6 & 0 & 18.640037 & -2.200694 & 1.209713 \\
\hline 33 & 1 & 0 & 17.277265 & -3.616602 & 2.108773 \\
\hline 34 & 6 & 0 & 21.221761 & -1.804200 & -0.112259 \\
\hline 35 & 6 & 0 & 20.915187 & -2.916976 & 0.696422 \\
\hline 36 & 6 & 0 & 22.285533 & -1.851332 & -1.012360 \\
\hline 37 & 6 & 0 & 21.711246 & -4.066024 & 0.575446 \\
\hline 38 & 6 & 0 & 23.090267 & -2.989086 & -1.098729 \\
\hline 39 & 1 & 0 & 22.489371 & -0.984272 & -1.634414 \\
\hline 40 & 1 & 0 & 21.483207 & -4.948833 & 1.162127 \\
\hline 41 & 6 & 0 & 22.798216 & -4.092505 & -0.300736 \\
\hline 42 & 1 & 0 & 23.929682 & -3.009926 & -1.787368 \\
\hline 43 & 1 & 0 & 23.405698 & -4.991069 & -0.364359 \\
\hline 44 & 16 & 0 & 20.292479 & -0.296498 & 0.090790 \\
\hline 45 & 7 & 0 & 19.831105 & -2.849549 & 1.603169 \\
\hline 46 & 6 & 0 & 19.754914 & -3.844948 & 2.660448 \\
\hline 47 & 1 & 0 & 19.102522 & -3.470803 & 3.453032 \\
\hline 48 & 1 & 0 & 19.370198 & -4.820496 & 2.323054 \\
\hline 49 & 1 & 0 & 20.752444 & -3.992670 & 3.080995 \\
\hline 50 & 6 & 0 & -0.968518 & 1.015651 & -0.593679 \\
\hline 51 & 6 & 0 & -0.717391 & -0.351655 & -0.819627 \\
\hline 52 & 6 & 0 & -2.305522 & 1.441122 & -0.455143 \\
\hline
\end{tabular}




\begin{tabular}{|c|c|c|c|c|c|}
\hline 53 & 6 & 0 & -1.753834 & -1.279076 & -0.907926 \\
\hline 54 & 1 & 0 & 0.307306 & -0.685470 & -0.955893 \\
\hline 55 & 6 & 0 & -3.342282 & 0.523760 & -0.543807 \\
\hline 56 & 1 & 0 & -2.512274 & 2.488844 & -0.250871 \\
\hline 57 & 6 & 0 & -3.072470 & -0.840717 & -0.770857 \\
\hline 58 & 1 & 0 & -1.529842 & -2.326936 & -1.091330 \\
\hline 59 & 6 & 0 & -4.846542 & 0.766866 & -0.412398 \\
\hline 60 & 6 & 0 & -4.344083 & -1.566859 & -0.814362 \\
\hline 61 & 6 & 0 & -5.392452 & -0.649070 & -0.602739 \\
\hline 62 & 6 & 0 & -4.629345 & -2.918216 & -1.019976 \\
\hline 63 & 6 & 0 & -6.712069 & -1.076419 & -0.597097 \\
\hline 64 & 6 & 0 & -5.957361 & -3.340783 & -1.011134 \\
\hline 65 & 1 & 0 & -3.831291 & -3.639923 & -1.175280 \\
\hline 66 & 6 & 0 & -7.017479 & -2.437467 & -0.802168 \\
\hline 67 & 1 & 0 & -7.521887 & -0.363590 & -0.462084 \\
\hline 68 & 1 & 0 & -6.180533 & -4.395971 & -1.140915 \\
\hline 69 & 6 & 0 & -5.361353 & 1.722632 & -1.511845 \\
\hline 70 & 1 & 0 & -6.449257 & 1.839450 & -1.445490 \\
\hline 71 & 1 & 0 & -4.909760 & 2.715625 & -1.403258 \\
\hline 72 & 1 & 0 & -5.118615 & 1.343469 & -2.509671 \\
\hline 73 & 6 & 0 & -5.207640 & 1.324851 & 0.982564 \\
\hline 74 & 1 & 0 & -4.850586 & 0.661718 & 1.776920 \\
\hline 75 & 1 & 0 & -4.757604 & 2.312983 & 1.133864 \\
\hline 76 & 1 & 0 & -6.293740 & 1.430462 & 1.087171 \\
\hline 77 & 6 & 0 & 8.750524 & 2.803531 & -0.716166 \\
\hline 78 & 6 & 0 & 9.507408 & 3.030892 & -1.882036 \\
\hline 79 & 6 & 0 & 9.340460 & 2.076634 & 0.338047 \\
\hline 80 & 6 & 0 & 10.811519 & 2.556825 & -2.009952 \\
\hline 81 & 1 & 0 & 9.052381 & 3.563969 & -2.711947 \\
\hline 82 & 6 & 0 & 10.637067 & 1.598886 & 0.216409 \\
\hline 83 & 1 & 0 & 8.778234 & 1.916678 & 1.254879 \\
\hline 84 & 6 & 0 & 11.379631 & 1.835898 & -0.957661 \\
\hline 85 & 1 & 0 & 11.368050 & 2.740020 & -2.925728 \\
\hline 86 & 6 & 0 & 11.461023 & 0.804105 & 1.230604 \\
\hline 87 & 6 & 0 & 12.700584 & 1.221015 & -0.806476 \\
\hline 88 & 6 & 0 & 12.767829 & 0.607618 & 0.460554 \\
\hline 89 & 6 & 0 & 13.798230 & 1.166938 & -1.667779 \\
\hline 90 & 6 & 0 & 13.919462 & -0.052833 & 0.862771 \\
\hline 91 & 6 & 0 & 14.950296 & 0.497938 & -1.258820 \\
\hline 92 & 1 & 0 & 13.759335 & 1.627580 & -2.651736 \\
\hline 93 & 6 & 0 & 15.035350 & -0.121027 & 0.003578 \\
\hline 94 & 1 & 0 & 13.980981 & -0.500050 & 1.851946 \\
\hline 95 & 1 & 0 & 15.793032 & 0.427255 & -1.940606 \\
\hline 96 & 6 & 0 & 10.793237 & -0.549074 & 1.562721 \\
\hline
\end{tabular}




\begin{tabular}{|c|c|c|c|c|c|}
\hline 97 & 1 & 0 & 11.425077 & -1.136868 & 2.238779 \\
\hline 98 & 1 & 0 & 9.827176 & -0.393476 & 2.056828 \\
\hline 99 & 1 & 0 & 10.622745 & -1.137767 & 0.655675 \\
\hline 100 & 6 & 0 & 11.687776 & 1.612825 & 2.527610 \\
\hline 101 & 1 & 0 & 12.160366 & 2.576589 & 2.313241 \\
\hline 102 & 1 & 0 & 10.735556 & 1.803812 & 3.036041 \\
\hline 103 & 1 & 0 & 12.334319 & 1.061063 & 3.219801 \\
\hline 104 & 6 & 0 & -8.423194 & -2.908667 & -0.799396 \\
\hline 105 & 6 & 0 & -8.862802 & -3.915678 & -1.670152 \\
\hline 106 & 6 & 0 & -9.373560 & -2.352984 & 0.074847 \\
\hline 107 & 6 & 0 & -10.187664 & -4.347928 & -1.675102 \\
\hline 108 & 1 & 0 & -8.170522 & -4.342603 & -2.389873 \\
\hline 109 & 6 & 0 & -10.689149 & -2.804602 & 0.101696 \\
\hline 110 & 1 & 0 & -9.072827 & -1.583376 & 0.779709 \\
\hline 111 & 6 & 0 & -11.126000 & -3.813107 & -0.779953 \\
\hline 112 & 1 & 0 & -10.490328 & -5.097843 & -2.397417 \\
\hline 113 & 6 & 0 & -13.318087 & -2.262615 & 0.388022 \\
\hline 114 & 6 & 0 & -13.502376 & -3.323037 & -0.521378 \\
\hline 115 & 6 & 0 & -14.326951 & -1.331506 & 0.613233 \\
\hline 116 & 6 & 0 & -14.737449 & -3.409629 & -1.180824 \\
\hline 117 & 6 & 0 & -15.576295 & -1.432586 & -0.023553 \\
\hline 118 & 1 & 0 & -14.150818 & -0.535925 & 1.331248 \\
\hline 119 & 1 & 0 & -14.907969 & -4.187144 & -1.917122 \\
\hline 120 & 6 & 0 & -15.755285 & -2.493469 & -0.922373 \\
\hline 121 & 1 & 0 & -16.690833 & -2.583973 & -1.466526 \\
\hline 122 & 6 & 0 & -12.795765 & -5.513446 & -1.393837 \\
\hline 123 & 1 & 0 & -12.896864 & -5.428194 & -2.487301 \\
\hline 124 & 1 & 0 & -12.013694 & -6.244044 & -1.174254 \\
\hline 125 & 1 & 0 & -13.737484 & -5.889389 & -0.986951 \\
\hline 126 & 7 & 0 & -12.465956 & -4.257666 & -0.738913 \\
\hline 127 & 16 & 0 & -11.812236 & -2.183200 & 1.339687 \\
\hline 128 & 6 & 0 & -16.653793 & -0.448946 & 0.240796 \\
\hline 129 & 6 & 0 & -16.352939 & 0.910998 & 0.449484 \\
\hline 130 & 6 & 0 & -18.003223 & -0.854126 & 0.285619 \\
\hline 131 & 6 & 0 & -17.352596 & 1.849834 & 0.697968 \\
\hline 132 & 1 & 0 & -15.319377 & 1.239677 & 0.388159 \\
\hline 133 & 6 & 0 & -19.003578 & 0.076016 & 0.528502 \\
\hline 134 & 1 & 0 & -18.247622 & -1.905448 & 0.155099 \\
\hline 135 & 6 & 0 & -18.684047 & 1.432155 & 0.736921 \\
\hline 136 & 1 & 0 & -17.090183 & 2.894080 & 0.848155 \\
\hline 137 & 6 & 0 & -20.513137 & -0.148486 & 0.626280 \\
\hline 138 & 6 & 0 & -19.926588 & 2.175389 & 0.972425 \\
\hline 139 & 6 & 0 & -21.005916 & 1.270542 & 0.911342 \\
\hline 140 & 6 & 0 & -20.148808 & 3.530937 & 1.224528 \\
\hline
\end{tabular}




$\begin{array}{lllrrr}141 & 6 & 0 & -22.307664 & 1.720380 & 1.101204 \\ 142 & 6 & 0 & -21.458895 & 3.976097 & 1.414263 \\ 143 & 1 & 0 & -19.319195 & 4.231869 & 1.272984 \\ 144 & 6 & 0 & -22.530823 & 3.079482 & 1.353445 \\ 145 & 1 & 0 & -23.148331 & 1.031673 & 1.056027 \\ 146 & 1 & 0 & -21.647157 & 5.028273 & 1.610974 \\ 147 & 1 & 0 & -23.544909 & 3.440433 & 1.503271 \\ 148 & 6 & 0 & -21.088651 & -0.689028 & -0.701932 \\ 149 & 1 & 0 & -20.848038 & -0.022173 & -1.536020 \\ 150 & 1 & 0 & -20.677936 & -1.680376 & -0.926303 \\ 151 & 1 & 0 & -22.179246 & -0.782815 & -0.642372 \\ 152 & 6 & 0 & -20.865206 & -1.110031 & 1.783585 \\ 153 & 1 & 0 & -20.451342 & -2.108004 & 1.597757 \\ 154 & 1 & 0 & -20.463751 & -0.745469 & 2.734440 \\ 155 & 1 & 0 & -21.951941 & -1.210385 & 1.886697\end{array}$

$\mathrm{HF}=-4599.0441711$

Table S9. Atom coordinates and total energies (in Hartrees) of optimized computed structure of $(\mathrm{FPTZ})_{4}$, according to B3LYP/6-31G ${ }^{*}$ calculations.

$\begin{array}{rrrrrr}1 & 6 & 0 & 6.761753 & -2.230594 & -0.531181 \\ 2 & 6 & 0 & 7.682582 & -3.150123 & -1.029737 \\ 3 & 6 & 0 & 8.983552 & -2.755233 & -1.375518 \\ 4 & 6 & 0 & 9.333502 & -1.405083 & -1.174226 \\ 5 & 6 & 0 & 8.420056 & -0.502279 & -0.639364 \\ 6 & 6 & 0 & 7.106396 & -0.888224 & -0.320164 \\ 7 & 6 & 0 & 11.880216 & -2.292340 & -1.373995 \\ 8 & 6 & 0 & 11.283132 & -3.557748 & -1.536339 \\ 9 & 6 & 0 & 12.082445 & -4.689584 & -1.317804 \\ 10 & 1 & 0 & 11.656609 & -5.682312 & -1.411614 \\ 11 & 6 & 0 & 13.430944 & -4.564036 & -0.989334 \\ 12 & 6 & 0 & 14.031748 & -3.307857 & -0.828372 \\ 13 & 6 & 0 & 13.218301 & -2.175781 & -1.009418 \\ 14 & 1 & 0 & 5.769253 & -2.578736 & -0.260336 \\ 15 & 1 & 0 & 7.382985 & -4.187107 & -1.133220 \\ 16 & 1 & 0 & 8.724257 & 0.532360 & -0.510164 \\ 17 & 1 & 0 & 14.027557 & -5.463679 & -0.870806 \\ 18 & 1 & 0 & 13.632891 & -1.184046 & -0.854638 \\ 19 & 16 & 0 & 10.928447 & -0.826218 & -1.722863 \\ 20 & 7 & 0 & 9.925303 & -3.658044 & -1.915105 \\ 21 & 6 & 0 & 9.445239 & -4.932075 & -2.426247 \\ 22 & 1 & 0 & 10.195158 & -5.347737 & -3.103296 \\ 23 & 1 & 0 & 9.235732 & -5.673630 & -1.639181 \\ 24 & 1 & 0.528169 & -4.763016 & -2.995628 \\ 25 & 6 & 0 & 23.573605 & -0.956721 & 0.141215\end{array}$




\begin{tabular}{|c|c|c|c|c|c|}
\hline 26 & 6 & 0 & 23.800149 & 0.421899 & 0.023100 \\
\hline 27 & 6 & 0 & 24.703025 & -1.791917 & 0.202932 \\
\hline 28 & 6 & 0 & 25.089791 & 0.947403 & -0.030060 \\
\hline 29 & 1 & 0 & 22.956464 & 1.105748 & 0.014861 \\
\hline 30 & 6 & 0 & 25.992808 & -1.278252 & 0.115003 \\
\hline 31 & 1 & 0 & 24.574954 & -2.867866 & 0.275906 \\
\hline 32 & 6 & 0 & 26.213600 & 0.108416 & -0.001734 \\
\hline 33 & 1 & 0 & 25.215333 & 2.023348 & -0.078821 \\
\hline 34 & 6 & 0 & 28.581575 & -1.370023 & 0.878773 \\
\hline 35 & 6 & 0 & 28.551362 & 0.026665 & 0.692851 \\
\hline 36 & 6 & 0 & 29.578133 & -1.962361 & 1.653385 \\
\hline 37 & 6 & 0 & 29.552557 & 0.799979 & 1.299611 \\
\hline 38 & 6 & 0 & 30.587200 & -1.184519 & 2.224700 \\
\hline 39 & 1 & 0 & 29.567876 & -3.039760 & 1.792689 \\
\hline 40 & 1 & 0 & 29.540676 & 1.879464 & 1.198991 \\
\hline 41 & 6 & 0 & 30.569348 & 0.196061 & 2.042151 \\
\hline 42 & 1 & 0 & 31.371649 & -1.656008 & 2.809175 \\
\hline 43 & 1 & 0 & 31.339462 & 0.818096 & 2.489915 \\
\hline 44 & 16 & 0 & 27.386124 & -2.388078 & 0.034614 \\
\hline 45 & 7 & 0 & 27.530803 & 0.608767 & -0.095021 \\
\hline 46 & 6 & 0 & 27.717693 & 1.971030 & -0.568178 \\
\hline 47 & 1 & 0 & 27.049803 & 2.146493 & -1.414835 \\
\hline 48 & 1 & 0 & 27.518936 & 2.737336 & 0.197814 \\
\hline 49 & 1 & 0 & 28.747088 & 2.087720 & -0.915414 \\
\hline 50 & 6 & 0 & 6.130840 & 0.087064 & 0.223079 \\
\hline 51 & 6 & 0 & 6.538828 & 1.089970 & 1.123758 \\
\hline 52 & 6 & 0 & 4.772653 & 0.029148 & -0.150314 \\
\hline 53 & 6 & 0 & 5.634965 & 2.013751 & 1.645412 \\
\hline 54 & 1 & 0 & 7.577606 & 1.126370 & 1.439550 \\
\hline 55 & 6 & 0 & 3.867416 & 0.944957 & 0.365350 \\
\hline 56 & 1 & 0 & 4.450022 & -0.722262 & -0.866900 \\
\hline 57 & 6 & 0 & 4.293002 & 1.941928 & 1.266053 \\
\hline 58 & 1 & 0 & 5.977676 & 2.772003 & 2.345044 \\
\hline 59 & 6 & 0 & 2.369200 & 1.068023 & 0.084372 \\
\hline 60 & 6 & 0 & 3.135857 & 2.761086 & 1.635749 \\
\hline 61 & 6 & 0 & 2.003996 & 2.272982 & 0.952366 \\
\hline 62 & 6 & 0 & 3.015442 & 3.856969 & 2.492693 \\
\hline 63 & 6 & 0 & 0.765660 & 2.874680 & 1.120775 \\
\hline 64 & 6 & 0 & 1.768511 & 4.458033 & 2.655389 \\
\hline 65 & 1 & 0 & 3.880420 & 4.250437 & 3.020785 \\
\hline 66 & 6 & 0 & 0.627549 & 3.984775 & 1.978815 \\
\hline 67 & 1 & 0 & -0.111895 & 2.480693 & 0.614027 \\
\hline 68 & 1 & 0 & 1.678190 & 5.330236 & 3.296703 \\
\hline 69 & 6 & 0 & 1.606898 & -0.199802 & 0.530604 \\
\hline
\end{tabular}




\begin{tabular}{|c|c|c|c|c|c|}
\hline 70 & 1 & 0 & 0.527723 & -0.074235 & 0.385006 \\
\hline 71 & 1 & 0 & 1.926306 & -1.069326 & -0.055681 \\
\hline 72 & 1 & 0 & 1.786316 & -0.415950 & 1.588637 \\
\hline 73 & 6 & 0 & 2.096531 & 1.348501 & -1.410382 \\
\hline 74 & 1 & 0 & 2.627315 & 2.244877 & -1.746564 \\
\hline 75 & 1 & 0 & 2.424169 & 0.504914 & -2.028869 \\
\hline 76 & 1 & 0 & 1.025047 & 1.498568 & -1.586587 \\
\hline 77 & 6 & 0 & 15.467362 & -3.173031 & -0.482473 \\
\hline 78 & 6 & 0 & 16.080365 & -4.073588 & 0.410180 \\
\hline 79 & 6 & 0 & 16.250489 & -2.145197 & -1.046028 \\
\hline 80 & 6 & 0 & 17.430724 & -3.970115 & 0.738813 \\
\hline 81 & 1 & 0 & 15.478586 & -4.851191 & 0.872130 \\
\hline 82 & 6 & 0 & 17.595246 & -2.036438 & -0.723657 \\
\hline 83 & 1 & 0 & 15.796666 & -1.459215 & -1.757183 \\
\hline 84 & 6 & 0 & 18.193040 & -2.947783 & 0.169832 \\
\hline 85 & 1 & 0 & 17.874849 & -4.675296 & 1.436943 \\
\hline 86 & 6 & 0 & 18.619208 & -1.020619 & -1.231909 \\
\hline 87 & 6 & 0 & 19.608587 & -2.598849 & 0.314284 \\
\hline 88 & 6 & 0 & 19.878875 & -1.476372 & -0.493934 \\
\hline 89 & 6 & 0 & 20.630793 & -3.178420 & 1.068578 \\
\hline 90 & 6 & 0 & 21.157904 & -0.942940 & -0.553268 \\
\hline 91 & 6 & 0 & 21.912102 & -2.633642 & 1.008396 \\
\hline 92 & 1 & 0 & 20.436615 & -4.038100 & 1.705039 \\
\hline 93 & 6 & 0 & 22.202020 & -1.516522 & 0.201006 \\
\hline 94 & 1 & 0 & 21.372765 & -0.098452 & -1.203398 \\
\hline 95 & 1 & 0 & 22.700978 & -3.064907 & 1.617981 \\
\hline 96 & 6 & 0 & 18.226200 & 0.421075 & -0.839136 \\
\hline 97 & 1 & 0 & 18.999965 & 1.131836 & -1.152047 \\
\hline 98 & 1 & 0 & 17.287119 & 0.711903 & -1.324144 \\
\hline 99 & 1 & 0 & 18.094883 & 0.513091 & 0.243749 \\
\hline 100 & 6 & 0 & 18.796798 & -1.119564 & -2.763778 \\
\hline 101 & 1 & 0 & 19.075462 & -2.134737 & -3.063630 \\
\hline 102 & 1 & 0 & 17.866799 & -0.853183 & -3.279225 \\
\hline 103 & 1 & 0 & 19.580091 & -0.434144 & -3.107695 \\
\hline 104 & 6 & 0 & -0.688456 & 4.642426 & 2.162301 \\
\hline 105 & 6 & 0 & -1.091892 & 5.148205 & 3.405968 \\
\hline 106 & 6 & 0 & -1.587086 & 4.777202 & 1.089593 \\
\hline 107 & 6 & 0 & -2.333700 & 5.757234 & 3.576781 \\
\hline 108 & 1 & 0 & -0.444657 & 5.027862 & 4.269732 \\
\hline 109 & 6 & 0 & -2.812443 & 5.417922 & 1.242949 \\
\hline 110 & 1 & 0 & -1.307829 & 4.415774 & 0.104199 \\
\hline 111 & 6 & 0 & -3.214426 & 5.919509 & 2.496926 \\
\hline 112 & 1 & 0 & -2.619732 & 6.093703 & 4.567001 \\
\hline 113 & 6 & 0 & -5.433086 & 5.611609 & 0.604590 \\
\hline
\end{tabular}




\begin{tabular}{|c|c|c|c|c|c|}
\hline 114 & 6 & 0 & -5.581942 & 6.081633 & 1.924220 \\
\hline 115 & 6 & 0 & -6.526288 & 5.133001 & -0.111135 \\
\hline 116 & 6 & 0 & -6.867499 & 6.053612 & 2.484814 \\
\hline 117 & 6 & 0 & -7.822038 & 5.139731 & 0.434138 \\
\hline 118 & 1 & 0 & -6.363811 & 4.740311 & -1.110455 \\
\hline 119 & 1 & 0 & -7.023789 & 6.387482 & 3.504481 \\
\hline 120 & 6 & 0 & -7.963601 & 5.609994 & 1.747121 \\
\hline 121 & 1 & 0 & -8.949159 & 5.645383 & 2.202045 \\
\hline 122 & 6 & 0 & -4.695442 & 7.366142 & 3.827425 \\
\hline 123 & 1 & 0 & -4.947396 & 6.767810 & 4.717234 \\
\hline 124 & 1 & 0 & -3.797858 & 7.949776 & 4.045077 \\
\hline 125 & 1 & 0 & -5.515382 & 8.061554 & 3.633455 \\
\hline 126 & 7 & 0 & -4.461431 & 6.567621 & 2.634550 \\
\hline 127 & 16 & 0 & -3.838277 & 5.713811 & -0.184956 \\
\hline 128 & 6 & 0 & -8.987435 & 4.666526 & -0.351192 \\
\hline 129 & 6 & 0 & -9.072342 & 4.916375 & -1.734727 \\
\hline 130 & 6 & 0 & -10.037858 & 3.961336 & 0.271064 \\
\hline 131 & 6 & 0 & -10.162366 & 4.485879 & -2.488853 \\
\hline 132 & 1 & 0 & -8.281932 & 5.482415 & -2.219396 \\
\hline 133 & 6 & 0 & -11.127423 & 3.532739 & -0.472671 \\
\hline 134 & 1 & 0 & -9.971962 & 3.735746 & 1.332620 \\
\hline 135 & 6 & 0 & -11.196095 & 3.792649 & -1.856153 \\
\hline 136 & 1 & 0 & -10.204114 & 4.701289 & -3.553603 \\
\hline 137 & 6 & 0 & -12.358748 & 2.756740 & -0.002671 \\
\hline 138 & 6 & 0 & -12.445532 & 3.230829 & -2.374935 \\
\hline 139 & 6 & 0 & -13.138049 & 2.617809 & -1.311495 \\
\hline 140 & 6 & 0 & -12.992834 & 3.227408 & -3.659435 \\
\hline 141 & 6 & 0 & -14.365535 & 2.009040 & -1.528354 \\
\hline 142 & 6 & 0 & -14.225461 & 2.612360 & -3.870368 \\
\hline 143 & 1 & 0 & -12.466623 & 3.687875 & -4.491886 \\
\hline 144 & 6 & 0 & -14.932511 & 1.996967 & -2.819173 \\
\hline 145 & 1 & 0 & -14.912667 & 1.562467 & -0.701636 \\
\hline 146 & 1 & 0 & -14.639785 & 2.582122 & -4.874084 \\
\hline 147 & 6 & 0 & -11.970065 & 1.375543 & 0.570900 \\
\hline 148 & 1 & 0 & -11.400419 & 0.791591 & -0.158957 \\
\hline 149 & 1 & 0 & -11.356038 & 1.489504 & 1.471822 \\
\hline 150 & 1 & 0 & -12.865009 & 0.804261 & 0.843563 \\
\hline 151 & 6 & 0 & -13.160680 & 3.556797 & 1.048059 \\
\hline 152 & 1 & 0 & -12.565260 & 3.707312 & 1.956122 \\
\hline 153 & 1 & 0 & -13.448165 & 4.539349 & 0.660465 \\
\hline 154 & 1 & 0 & -14.073549 & 3.019384 & 1.329869 \\
\hline 155 & 6 & 0 & -16.245288 & 1.354000 & -3.066553 \\
\hline 156 & 6 & 0 & -17.170568 & 1.897649 & -3.968678 \\
\hline 157 & 6 & 0 & -16.615239 & 0.175201 & -2.395661 \\
\hline
\end{tabular}




\begin{tabular}{|c|c|c|c|c|c|}
\hline 158 & 6 & 0 & -18.409636 & 1.299973 & -4.190900 \\
\hline 159 & 1 & 0 & -16.940318 & 2.829451 & -4.476818 \\
\hline 160 & 6 & 0 & -17.834733 & -0.448349 & -2.639928 \\
\hline 161 & 1 & 0 & -15.921249 & -0.290716 & -1.702170 \\
\hline 162 & 6 & 0 & -18.762859 & 0.106956 & -3.543193 \\
\hline 163 & 1 & 0 & -19.109680 & 1.781413 & -4.864638 \\
\hline 164 & 6 & 0 & -19.950627 & -1.847320 & -1.711827 \\
\hline 165 & 6 & 0 & -20.675225 & -1.153275 & -2.701014 \\
\hline 166 & 6 & 0 & -20.603078 & -2.463222 & -0.648574 \\
\hline 167 & 6 & 0 & -22.071495 & -1.107992 & -2.575507 \\
\hline 168 & 6 & 0 & -22.004643 & -2.448454 & -0.537939 \\
\hline 169 & 1 & 0 & -20.009879 & -3.002101 & 0.084329 \\
\hline 170 & 1 & 0 & -22.663918 & -0.549701 & -3.291790 \\
\hline 171 & 6 & 0 & -22.719275 & -1.755982 & -1.525600 \\
\hline 172 & 1 & 0 & -23.800384 & -1.682585 & -1.453677 \\
\hline 173 & 6 & 0 & -20.767338 & -0.153238 & -4.947961 \\
\hline 174 & 1 & 0 & -21.328388 & 0.785671 & -4.818113 \\
\hline 175 & 1 & 0 & -20.089141 & -0.037059 & -5.796637 \\
\hline 176 & 1 & 0 & -21.475894 & -0.950498 & -5.184528 \\
\hline 177 & 7 & 0 & -19.996612 & -0.539202 & -3.776632 \\
\hline 178 & 16 & 0 & -18.185828 & -2.028855 & -1.892206 \\
\hline 179 & 6 & 0 & -22.693706 & -3.133578 & 0.582021 \\
\hline 180 & 6 & 0 & -22.123789 & -3.166188 & 1.869561 \\
\hline 181 & 6 & 0 & -23.936782 & -3.767659 & 0.380966 \\
\hline 182 & 6 & 0 & -22.756914 & -3.808534 & 2.932155 \\
\hline 183 & 1 & 0 & -21.181133 & -2.655488 & 2.044358 \\
\hline 184 & 6 & 0 & -24.574782 & -4.404346 & 1.435502 \\
\hline 185 & 1 & 0 & -24.375806 & -3.776283 & -0.613662 \\
\hline 186 & 6 & 0 & -23.987905 & -4.430393 & 2.715802 \\
\hline 187 & 1 & 0 & -22.295141 & -3.811272 & 3.916353 \\
\hline 188 & 6 & 0 & -25.909483 & -5.150326 & 1.433206 \\
\hline 189 & 6 & 0 & -24.872364 & -5.171543 & 3.621471 \\
\hline 190 & 6 & 0 & -25.999546 & -5.600862 & 2.891512 \\
\hline 191 & 6 & 0 & -24.747339 & -5.473906 & 4.979219 \\
\hline 192 & 6 & 0 & -27.002094 & -6.332160 & 3.518408 \\
\hline 193 & 6 & 0 & -25.758030 & -6.208985 & 5.602650 \\
\hline 194 & 1 & 0 & -23.879641 & -5.144753 & 5.545542 \\
\hline 195 & 6 & 0 & -26.876993 & -6.635459 & 4.879667 \\
\hline 196 & 1 & 0 & -27.876452 & -6.668588 & 2.965691 \\
\hline 197 & 1 & 0 & -25.674353 & -6.451864 & 6.658688 \\
\hline 198 & 1 & 0 & -27.655524 & -7.206756 & 5.378270 \\
\hline 199 & 6 & 0 & -25.880163 & -6.357745 & 0.469670 \\
\hline 200 & 1 & 0 & -25.750459 & -6.023705 & -0.566354 \\
\hline 201 & 1 & 0 & -25.058219 & -7.037753 & 0.714860 \\
\hline
\end{tabular}




$\begin{array}{llllll}202 & 1 & 0 & -26.819014 & -6.920682 & 0.526349 \\ 203 & 6 & 0 & -27.077622 & -4.208799 & 1.062386 \\ 204 & 1 & 0 & -27.116712 & -3.345441 & 1.734337 \\ 205 & 1 & 0 & -26.966248 & -3.839536 & 0.036137 \\ 206 & 1 & 0 & -28.036283 & -4.736522 & 1.127404 \\ \mathrm{HF}=-6131.6619019 & & & & \end{array}$

